Research

\title{
Insecticide resistance profiles for malaria vectors in the Kassena-Nankana district of Ghana
} Francis Anto*1, Victor Asoala1, Thomas Anyorigiya1, Abraham Oduro1, Martin Adjuik ${ }^{1}$, Seth Owusu-Agyei ${ }^{2}$, Dominic Dery ${ }^{2}$, Langbong Bimi ${ }^{3}$ and Abraham Hodgson ${ }^{1}$

\author{
Address: ${ }^{1}$ Navrongo Health Research Centre, Navrongo Health Research Centre, Ghana Health Service, P. O. Box 114, Navrongo, Upper East \\ Region, Ghana, ${ }^{2}$ Kintampo Health Research Centre, P. O Box 200, Kintampo, Brong Ahafo Region, Ghana and ${ }^{3}$ Department of Zoology, University \\ of Ghana, Legon, Ghana \\ Email: Francis Anto* - franciskanto@yahoo.com; Victor Asoala - vasoala@navrongo.mimcom.net; \\ Thomas Anyorigiya - tanyorigiya@navrongo.mimcom.net; Abraham Oduro - aoduro@navrongo.mimcom.net; \\ Martin Adjuik - madjuik@navrongo.mimcom.net; Seth Owusu-Agyei - seth.owusu-agyei@ghana-khrc.org; \\ Dominic Dery - dominic.dery@ghana-khrc.org; Langbong Bimi - lbimi@ug.edu.gh; Abraham Hodgson - ahodgson@navrongo.mimcom.net \\ * Corresponding author
}

Published: 23 April 2009

Malaria Journal 2009, 8:8I doi:I0.1 I86/I475-2875-8-8I
Received: 17 November 2008

Accepted: 23 April 2009

This article is available from: http://www.malariajournal.com/content/8/l/81

(c) 2009 Anto et al; licensee BioMed Central Ltd.

This is an Open Access article distributed under the terms of the Creative Commons Attribution License (http://creativecommons.org/licenses/by/2.0), which permits unrestricted use, distribution, and reproduction in any medium, provided the original work is properly cited.

\begin{abstract}
Background: Malaria is a major public health problem in Ghana. The current strategy of the National Malaria Control Programme is based on effective case management and the use of insecticide treated bed nets among vulnerable groups such as children under-five years of age and pregnant women. Resistance to pyrethroids by Anopheles gambiae s.l. and Anopheles funestus has been reported in several African countries including neighbouring Burkina Faso.
\end{abstract}

Methods: Indoor resting Anopheles mosquitoes were collected. Blood-fed and gravid females were allowed to oviposit, eggs hatched and larvae reared to I-3 days old adults and tested against permethrin $0.75 \%$, deltamethrin $0.05 \%$, cyfluthrin $0.15 \%$, lambdacyhalothrin $0.1 \%$ and DDT $4 \%$, based on WHO methodology. PCR analyses were carried out on a subsample of 192 of the An. gambiae for sibling species complex determination. Resistance to pyrethroids and DDT was determined by genotyping the knock-down resistance kdr gene mutations in the study area.

Results: A total of 9,749 I-3 days-old FI female Anopheles mosquitoes were exposed to the insecticides. Among the pyrethroids, permethrin, $0.75 \%$ had the least knockdown effect, whilst cyfluthrin $0.15 \%$, had the highest knock-down effect. Overall, no difference in susceptibility between An. gambiae $93.3 \%$ (95\% Cl: $92.5-94.1$ ) and An. funestus $94.5 \%$ (95\% Cl: 93.7-95.3) was observed when exposed to the pyrethroids. Similarly, there was no difference in susceptibility between the two vector species (An. gambiae $=79.1 \%$ (95\% Cl: 76.6-8I.8) and An. funestus $=83.5 \%$ (95\% Cl: 80.2-86.4) when exposed to DDT. Overall susceptibility to the insecticides was between $80 \%$ and $98 \%$, suggesting that there is some level of resistance, except for cyfluthrin $0.15 \%$. The $\mathrm{kdr}$ PCR assay however, did not reveal any kdr mutations. The analysis also revealed only the molecular M (Mopti) form.

Conclusion: The findings in this study show that An. gambiae and An. funestus, the main malaria vector mosquitoes in the Kassena-Nankana district are susceptible to the insecticides being used in the treatment of bed nets in the malaria control programme. There is however, the need for continuous monitoring of the pyrethroids as the efficacy is not very high. 


\section{Background}

Malaria is a major public health problem in Ghana. The strategy of the National Malaria Control Programme is based on effective case management and the use of insecticide treated bed nets among vulnerable groups, such as children under five years of age and pregnant women.

There is renewed interest in the use of insecticides for malaria control because of the effectiveness of insecticidetreated materials that show promise in reducing malaria transmission and morbidity [1-3]. Insecticide-treated bed nets (ITN) have been used successfully in the KassenaNankana district of Ghana for over a decade now; first as the earliest experimental intervention trial followed by routine use among most community members. This led to the adoption of ITNs as national malaria control policy in support of the Roll Back Malaria (RBM) control programme.

The recent appearance of resistance in the malaria vector mosquitoes to the insecticides used for the treatment of bed nets in nearby countries is however a cause of concern in Ghana as this could impact negatively on the success of the current ITN programme. This is because vector susceptibility is a basic requirement for the efficacy of insecticides and for that matter the success of the RBM programme. Due to all year round irrigated agricultural activity and ITN use in the Kassena-Nankana district, it is logical that insect/vector resistance to the commonly used insecticides will develop with time. At present the insecticides for net-treatment are limited to the pyrethroids, as these are the only ones available and affordable. Any Anopheles strains that become resistant to any products of the compound often become cross-resistant to the others. Despite limited monitoring activities, resistances are already reported in a number of malaria vectors including some populations of Anopheles gambiae in Africa. The levels of resistance and impact on malaria control are not yet known.

The use of the pyrethroid insecticides is also spreading fast in public health due to its success stories in house-spraying, ITNs, insecticide-treated curtains, space spraying among others. Part of the debilitating effects of the wide use of pyrethroids is cross-resistance resulting from pyrethroids formulated for use in agriculture. This underscores the need to generate insecticide resistance related data that will be necessary to guide planning, implementation and evaluation of insecticides and their role in the control of malaria in Ghana.

The current study was to determine the resistance status of An. gambiae and Anopheles funestus, the main malaria vectors in the Kassena-Nankana district of northern Ghana. The assessment was carried out through the application of
WHO Bioassay tests on the vectors and determination of any knock down resistance $(k d r)$ genes that the vectors would have developed over the years. Investigations concentrated on four pyrethroids (permethrin, deltamethrin, lambdacyhalothrin and cyfluthrin) and DDT.

\section{Methods \\ Study sites}

The study was carried out in the Kassena Nankana District (KND) in northern Ghana. The district lies between $10^{\circ} 30^{\prime}$ and $11^{\circ} 00^{\prime} \mathrm{N}, 1^{\circ} 00^{\prime}$ and $1^{\circ} 30^{\prime} \mathrm{W}$ and borders Burkina Faso to the north. The size of the district is about $1,674 \mathrm{sq} \mathrm{km}$ of Sahelian savannah with a population of 143,000 (NDSS, 2006). This is the area, where a largescale insecticide treated bed net trial was undertaken about a decade ago, and has high malaria attributable infant and child mortality rate [3].

Most of the people live in multi-family compounds, which form the basis of the address system used in the Navrongo Demographic Surveillance System (NDSS, 2006), and are separated from one another by agricultural land. Virtually all the inhabitants engage in subsistence farming of millet, groundnut, and livestock. The average annual rainfall is $850 \mathrm{~mm}$, almost all of which occurs in the months of May to September, with the rest of the year being relatively dry. A large reservoir (Tono dam) in the middle of the district and about 90 dug-out dams provide water throughout the year for agricultural irrigation purposes.

\section{Field procedures}

Mosquitoes were collected from all the ecological areas (irrigated, low land and rocky highland) in the district. Indoor resting mosquitoes were collected using hand held aspirators and kept in paper cups covered with mesh. Moist cotton balls were added to the collection cups to increase survival of the samples. The blood fed and gravid females An. gambiae and An. funestus were allowed to oviposit and the larvae bred into adults in Navrongo Health Research Centre insectary.

\section{Mosquito identification}

Female Anopheles mosquitoes obtained in the field or reared in the laboratory were identified by morphological characteristics using the criteria of Gilles and de Meillon [4]. Mosquito species collected from the field were maintained in the insectary at $26^{\circ} \mathrm{C}$ and $80 \%$ Relative Humidity (RH). The $80 \%$ RH was maintained using a humidifier. Insecticide assays were conducted in a separate laboratory isolated from insect rearing areas. 


\section{Laboratory procedures \\ WHO susceptibility tests}

Laboratory-bred live mosquitoes were held for up to four hours before testing and supplied with cotton balls soaked with $10 \%$ sucrose solution. Susceptibility tests were performed using WHO test kits for measuring insecticide resistance. The insecticides tested were: permethrin $0.75 \%$, deltamethrin $0.05 \%$, cyfluthrin $0.15 \%$, lambdacyhalothrin $0.1 \%$ and DDT $4 \%$. Bioassays were done according to standard WHO methodology [5]. Female An. gambiae and An. funestus mosquitoes were exposed to insecticide-impregnated filter papers. Susceptibility tests were conducted using 1-3 days-old unfed female mosquitoes by exposing groups of 20-25 to established discriminating concentrations of each insecticide. Each experiment consisted of three replicates of the test and one control (filter paper without insecticide). The mosquitoes were exposed for a period of one hour with the assay cylinders in a vertical position. The number of mosquitoes knocked down after 10, 15, 20, 30 and 60 minutes were recorded. After one hour, the mosquitoes were transferred into holding containers and provided with cotton pads with $10 \%$ sucrose solution. Mortality was recorded after a 24-hour recovery period.

\section{Molecular forms and $\mathrm{kdr}$ analysis}

PCR analysis was carried out on a sub-sample each of the An. gambiae and An. funestus mosquitoes for sibling species complex determination. Resistance to pyrethroids was determined by genotyping the knock down resistance $k d r$ gene mutations [6] in the study area. One leg of a single mosquito was placed in a sterile $1.5 \mathrm{ml}$ micro-centrifuge tube and crushed in $15 \mu$ of double distilled water. The homogenized leg was then centrifuged at 14,000 RPM for 1 minute. DNA extract (supernatant) was then used immediately in the PCR mixture for the kdr gene identification. The PCR mixture consisted of an initial volume of $15.475 \mu \mathrm{l}$ of double distilled water. To provide nucleotides to the DNA strand during extension, $0.1 \mu \mathrm{l}$ of each of four $10 \mathrm{mM}$ dNTPs (deoxyribonucleoside triphosphate) was added to the double distilled water in the tube. These include dTTP, dCTP, dGTP, dATP. Primers Agd1, Agd2, Agd3 and Agd4 were then added followed by a PCR buffer containing $\mathrm{MgCl}_{2}$. The final reagent added was $0.125 \mu \mathrm{l}$ of Taq polymerase. $0.5 \mu \mathrm{l}$ of the DNA extract was then pipetted into a $0.2 \mathrm{ml}$ microtube followed by $19.6 \mu \mathrm{l}$ of the PCR mixture and then placed in a Thermal Cycler, PTC-0100 to run for about three and a half hours for amplification. The amplified products were then analysed by electrophoresis on 1.5\% agarose gel and visualized by ethidium bromide staining under UV light.

\section{Analysis of data}

All data were entered into a computer database at the Navrongo Health Research Centre. Results of all resistance tests were analysed for knock-down/time relationships. Analyses produced time-mortality data, which is a highly effective measure of insecticide resistance. Comparisons in terms of efficacy of the insecticides as well as susceptibility of the two vector species were made using 95\% confidence intervals. The genotyped results were used to interpret the pyrethroid resistance levels. Data used for the analysis are for bioassays that all the control mosquitoes exposed to untreated papers survived and so no corrections were made using Abbott's formula.

\section{Ethical issues}

The protocol was submitted to both the Navrongo Health Research Centre and Ghana Health Service ethical review committees for ethical consideration and a waiver was given. Verbal consent was sought from compound/household heads before in-door resting mosquitoes were collected from peoples' homes.

\section{Results \\ WHO susceptibility tests}

A total of about 9,749, 1-3 day-old F1 female Anopheles mosquitoes (Anopheles gambiae, 5,676 and An. funestus 4,073 ) were exposed to established discriminating concentrations of pyrethroid insecticides (permethrin $0.75 \%$, deltamethrin $0.05 \%$, cyfluthrin $0.15 \%$, and lambdacyhalothrin $0.1 \%$ ) and DDT (4\%) under laboratory conditions to determine their level of susceptibility. Over $60 \%$ of the malaria vector mosquitoes exposed was knocked down within an hour of exposure to the chemicals.

Among the pyrethroids, permethrin, $0.75 \%$ had the least knock-down effect, $63.2 \%$ and $65.0 \%$ respectively for $A n$. gambiae and An. funestus, whilst cyfluthrin $0.15 \%$, had the highest knock-down effect (Table 1). The knock-down effect of cyfluthrin was significantly higher than the other three pyrethroids, whilst deltamethrin $0.05 \%$, and lambdacyhalothrin $0.1 \%$ ) were more effective than permethrin (Figures 1 and 2). Deltamethrin 0.05\%, appeared to be slightly more potent (95.8\%; CI: 94.9-96.6) than lambdacyhalothrin $0.1 \%(93.6 \%$; CI: 92.2-94.8) leading to slightly more mortalities in exposed An. gambiae and An. funestus. Overall, no difference in susceptibility between An. gambiae (93.3\%; CI: 92.5-94.1) and An. funestus (94.5\%; CI: 93.7-95.3) was observed when exposed to the pyrethroids. Similarly, the difference in susceptibility between the two vector species (An. gambiae $=79.1 \%$; CI: 76.6-81.8 and An. funestus = 83.5\%; CI: 80.2-86.4) was not statistically significant when exposed to DDT. The pyrethroids had a higher knocked-down effect and caused a higher level of mortality in the mosquitoes than DDT $4 \%$ which started knocking down the mosquitoes only after 20 minutes of exposure (Figures 1 and 2). 


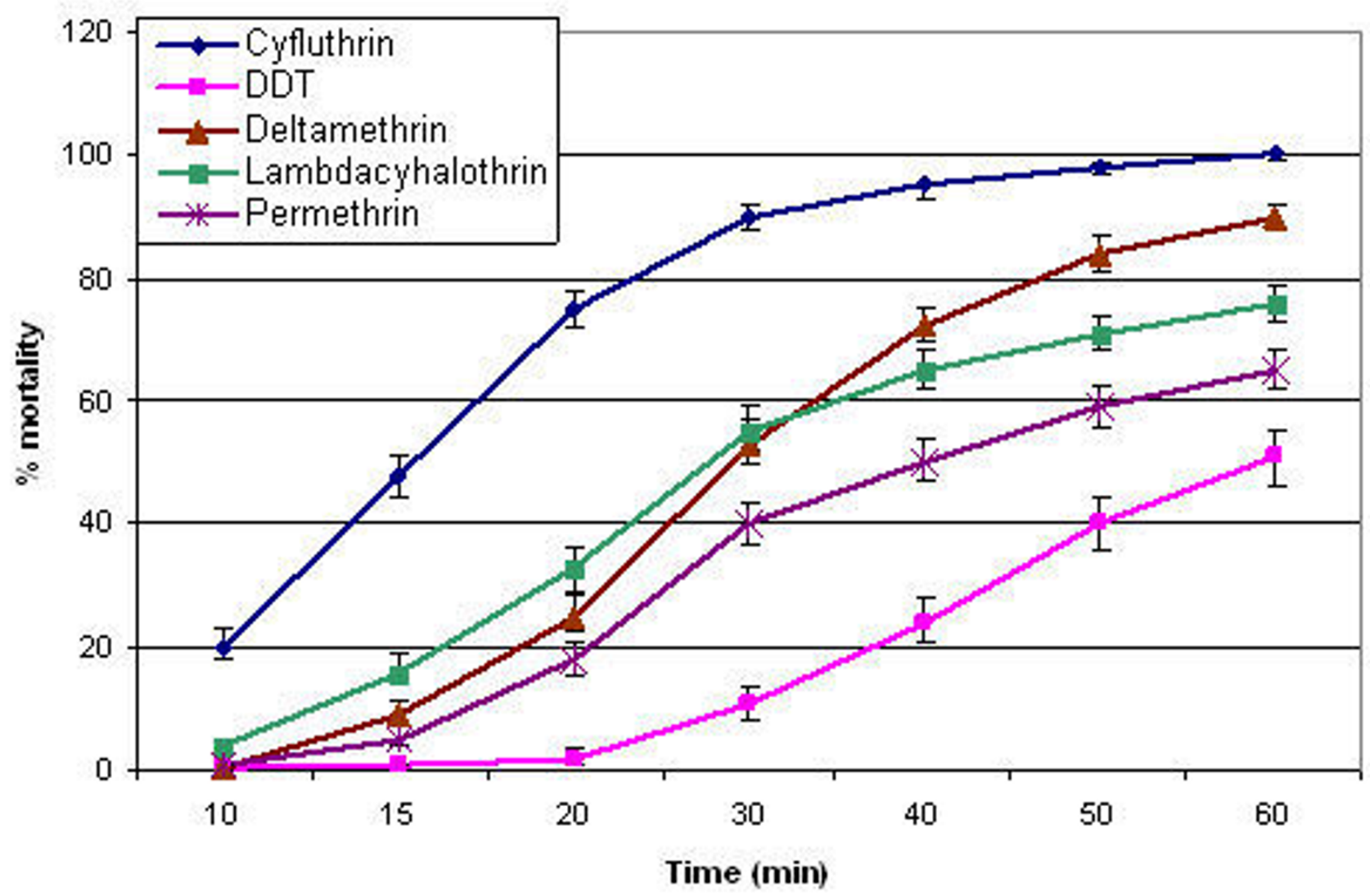

Figure I

Susceptibility of Anopheles funestus from the Kassena-Nankana district of northern Ghana to some pyrethroids and DDT.

\section{Susceptibility of mosquitoes collected from different ecological zones}

The overall susceptibility of the malaria mosquitoes vectors collected from the different ecological areas to the insecticides Permethrin 0.75\%, Deltamethrin 0.05\%, Lambdacyhalothrin $0.1 \%$ and DDT $4.0 \%$ was between 80 and $98 \%$. Susceptibility to Cyfluthrin however was much higher (Table 2).

\section{Seasonal variation in susceptibility of An. gambiae and} An. funestus mosquitoes to selected pyrethroid insecticides and DDT

During the dry season, a total of 4,710 mosquitoes vectors (An. funestus and An. gambiae were exposed to the insecticides. Out this number, 4,432 (94.1\%; CI: 93.4-94.8) were susceptible. A total of 5,039 were exposed during wet season with 4,527 (89.8\%; CI: 89.0-90.7) being susceptible. Thus, overall, there was a significant seasonal variation in susceptibility. Analysis at the species level also revealed significant seasonal variation in susceptibility. For An. funestus, dry season susceptibility was $90.5 \%$ (CI: 90.5-92.9) and that for the wet season was $87.3 \%$ (CI:
85.7-88.7). In the case of An. gambiae, susceptibility during the dry season was $89.8 \%$ (CI: 88.5-91.0) and the wet season was $91.4 \%$ (CI: 90.4-92.4).

\section{Molecular forms and $\mathbf{k d r}$}

After allowing for a 24 -hour recovery period, over $80 \%$ of the mosquitoes exposed died (Figure 3); mortality was however less than $98 \%$ (a level of mortality suggesting the possibility of resistance) except for cyfluthrin $0.15 \%$. The $k d r$ PCR assay of 192 of the samples (148 survivors (DDT: 67; deltamethrin: 17; permethrin: 64: and 44 susceptible ones) however, did not reveal any $k d r$ mutations in the mosquitoes. The analysis also revealed only the molecular $\mathrm{M}$ (Mopti) form.

\section{Discussion}

Female An. gambiae and An. funestus mosquitoes were collected from three micro-ecological areas (irrigated, low land and rocky highland) during the months of November 2006 to April 2007 (dry season) and July to October 2007 (wet season) in the Kassena-Nankana district of northern Ghana. These were bred in the laboratory and 


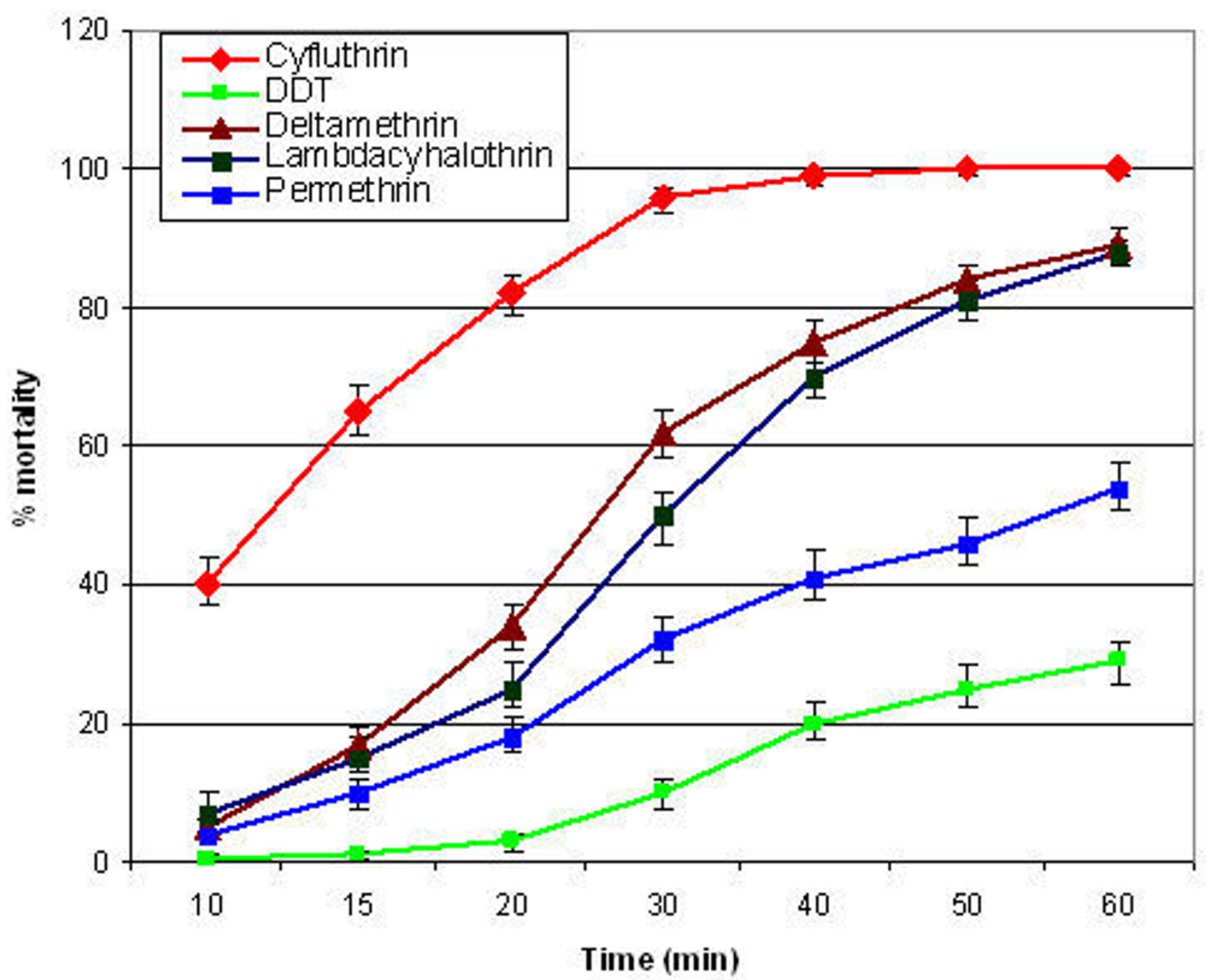

Figure 2

The knock-down effect of some pyrethroids and DDT on Anopheles gambiae from the Kassena-Nankana district of Northern Ghana.

Table I: Susceptibility of adult female Anopheles gambiae and An. funestus mosquitoes to selected pyrethroid insecticides and DDT

\begin{tabular}{lllll}
\hline & \multicolumn{2}{l}{ Number of mosquitoes tested } & \% knock-down after I hour exposure \\
\cline { 2 - 5 } Insecticides & An. gambiae & An. funestus & An. gambiae & An. funestus \\
\hline Permethrin $0.75 \%$ & 1372 & 881 & 63.2 & 65.0 \\
Deltamethrin $0.05 \%$ & 1451 & 1011 & 89.5 & 92.1 \\
Cyfluthrin 0.15\% & 897 & 854 & 99.6 & 99.8 \\
Lambdacyhalothrin 0.1\% & 888 & 758 & 88.2 & 94.4 \\
DDT 4\% & 1068 & 569 & 33.8 & 50.6
\end{tabular}


Table 2: Mortality of adult female malaria mosquitoes vectors collected from different ecological areas of the Kassena-Nankana district when exposed to different insecticides

\begin{tabular}{lllllll}
\hline & \multicolumn{3}{c}{ Number of mosquitoes tested } & & $\%$ mortality \\
\cline { 2 - 7 } Insecticide & Irrigated & Lowland & Rocky highland & Irrigated & Lowland & Rocky highland \\
\hline Permethrin 0.75\% & 887 & 534 & 832 & 94.6 & 85.3 & 82.4 \\
Deltamethrin 0.05\% & 1125 & 510 & 818 & 96.3 & 97.6 & 94.0 \\
Cyfluthrin 0.15\% & 897 & 506 & 348 & 100 & 100 & 99.8 \\
Lambda-cyhalothrin 0.1\% & 886 & 422 & 338 & 95.1 & 98.5 & 89.4 \\
DDT 4\% & 765 & 512 & 360 & 82.7 & 77.5 & 85.9 \\
\hline
\end{tabular}

the F1 progeny exposed to deltamethrin $0.05 \%$, lambdacyhalothrin $0.1 \%$, permethrin $0.75 \%$, cyflutrin $0.15 \%$, and DDT $4 \%$. Analysis of the results revealed that An. gambiae and An. funestus in the district are susceptible to the tested insecticides despite the over a decade of use of permethrin and deltamethrin in the treatment of bed nets in the district. Overall, between $80 \%$ and $98 \%$ of the exposed mosquitoes died during the 24-hour recovery period.
Susceptibility of An. gambiae collected from the low land and rocky highland areas was however less than $80 \%$, suggesting resistance in this malaria vector species to DDT, an insecticide that was in common use in the 1950s and 60s. Resistance in Anopheles gambiae to pyrethroid insecticides has mainly been associated with reduced target site sensitivity arising from a single point mutation in the sodium channel gene, often referred to as knockdown resistance $(k d r)$. PCR analysis of both samples that survived the sensitivity test and those that died however did not reveal the presence of the kdr gene [7]. Possibly, other mechanisms

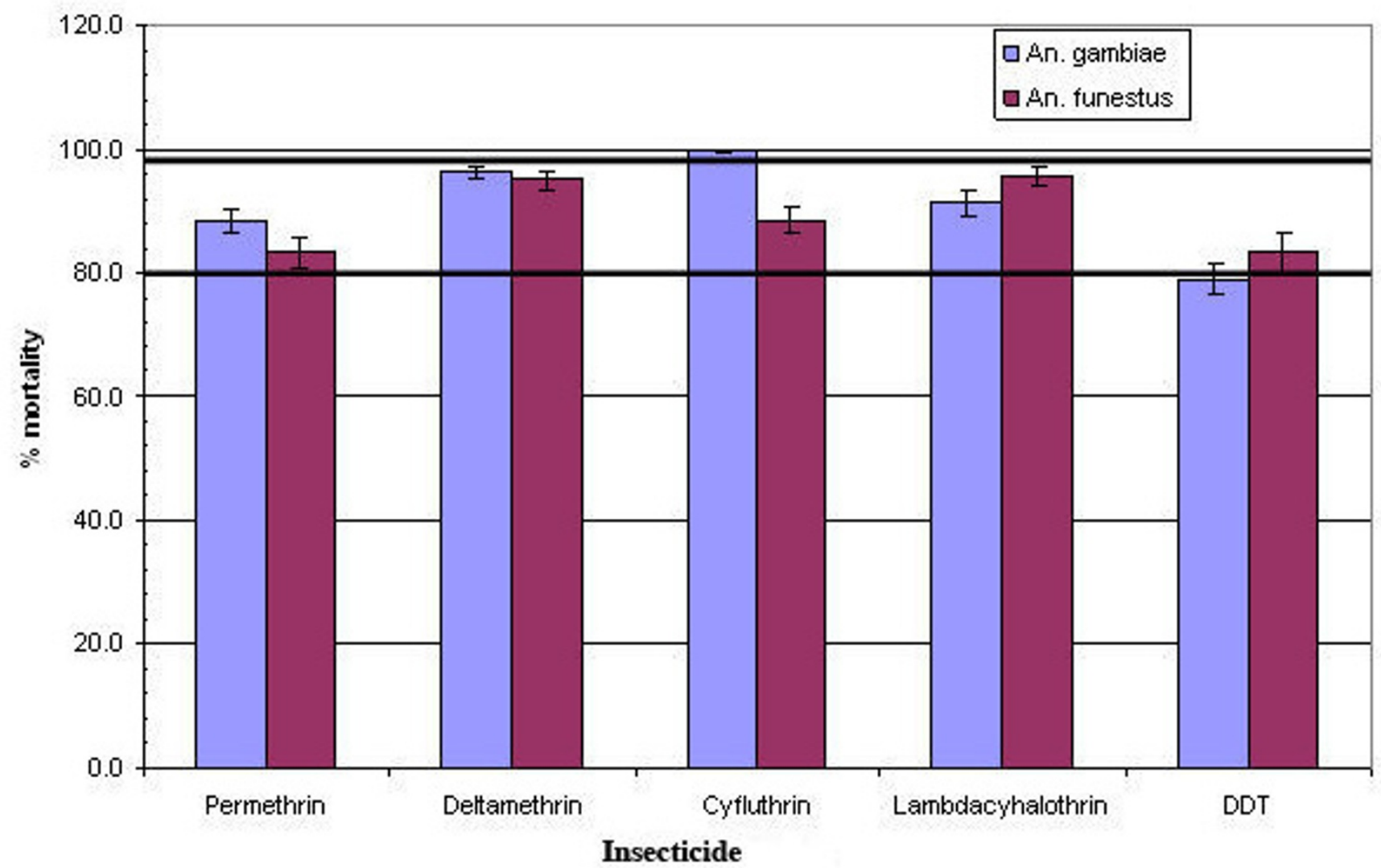

Figure 3

Susceptibility of Anopheles gambiae and Anopheles funestus to pyrethroids and DDT. 
of resistance e.g. metabolic detoxification might be playing a role in the apparent resistance of An. gambiae collected from these micro-ecological areas. The knock down rate of DDT was also found to be slow as mosquitoes started getting knocked-down only after 20 minutes of exposure $[8,9]$.

The low efficacy of permethrin and lambdacyhalothrin can be explained in terms of the extensive domestic and agricultural use of these two insecticides in the district $[10,11]$. There is also evidence of cross-resistance between DDT and the pyrethroids in An. gambiae [11], which can explain the low level of efficacy of both DDT and permethrin in our study area. Though DDT is no more in common use in the area, permethrin and other pyrethroids have been in use for a long time in agriculture for the control of pests and in the health sector for malaria control [3].

Anopheles funestus in the current study was found to be more susceptible to the insecticides during the dry season than during the wet season. Such seasonal variations in susceptibility of Anopheles mosquitoes to pyrethroids and DDT have been reported in neighbouring Burkina Faso. The increase in resistance of An. gambiae complex to permethrin $1 \%$, DDT $4 \%$ and deltamthrin $0.05 \%$ in the wet season was explained in terms of increased use of insecticides on cotton farms resulting in selection pressure on the mosquito population [10]. In addition to selection pressure due to increased use of insecticides, the harsh dry season in the Kassena-Nankana district and neighbouring Burkina Faso may impact on the physiology of the insects and increase mortality when exposed to given concentrations of insecticides during that period of the year.

The $\mathrm{M}$ and $\mathrm{S}$ forms of the An. gambiae complex have been reported to live sympatrically in the Kassena-Nankana district [12]. This study however, found only the molecular $\mathrm{M}$ form of An. gambiae s.s. and without the $k d r$ mutation. A study by Diabate and colleagues in neighbouring Burkina Faso also did not reveal any kdr mutation in this form of An. gambiae [10]. The dominance of the molecular $\mathrm{M}$ form however seems to correlate with ecological or climatic factors in the study area as the $\mathrm{M}$ form is more adapted to dryer environment and breeds along irrigated fields while the S form is normally found in humid forested areas and temporary pools [13]. The presence of the $\mathrm{S}$ and $\mathrm{M}$ forms is also known to change with the seasons [10].

Resistance of An. gambiae in West Africa to DDT has been known for a long time now $[14,15]$. It is expected, however, that after a long period of discontinuation of use, the efficacy will improve and could be used for indoor residual spraying since in costs less and has longer residual effect than the pyrethroids. The efficacy at the moment however, may not be good enough for use in a malaria control programme. The resistance of Anopheles species to the pyrethroids, the main insecticide being used by malaria control programmes for the treatment of bed nets has been reported in a number of African countries including Benin, Cote d'Ivoire, Burkina Faso, Kenya, Nigeria, Cameroun and Equatorial Guinea [9,10,16-18] $22[19,20]$.

The findings in this study show that An. gambiae and An. funestus, the main malaria mosquitoes vectors in the Kassena-Nankana district [12] are susceptible to the insecticides being used in the treatment of bed nets in the malaria control programme. There is however, the need for continuous monitoring of the pyrethroids as the efficacy is not very high. This also holds for DDT.

\section{Competing interests}

The authors declare that they have no competing interests.

\section{Authors' contributions}

FA, VA, TA, AO and SOA designed the study, developed the instruments, took part in data analysis and manuscript production. AH contributed to the development of the protocol. DD contributed to the laboratory analysis of samples. MA took part in the data analysis. LB contributed to producing the Manuscript.

\section{Acknowledgements}

We are very grateful to all individuals and groups who contributed to making this study a successful one. In particular, we are most grateful to the community members especially those individuals from whose rooms indoor resting mosquitoes were collected. We also acknowledge with gratitude the significant contributions made by Messrs Edmond Tampugre and Patrick Aberinga towards the successful completion of this project. This study was carried out with financial assistance from the Ghanaian-Dutch Collaboration for Health Research and Development and we are most grateful for that.

\section{References}

I. Greenwood BM, Pickering H: A malaria control trial using insecticide-treated bed nets and targeted chemoprophylaxis in a rural area of The Gambia, West Africa. I. A review of the epidemiology and control of malaria in the Gambia, West Africa. Trans R Soc Trop Med Hyg 1993, 87:3-II.

2. Choi HW, Breman JG, Teutsch SM, Liu S, Hightower AW, Sexton JD The effectiveness of insecticide-impregnated bed nets in reducing cases of malaria infection: a meta-analysis of published results. Am J Trop Med Hyg 1995, 52:377-382.

3. Binka FN, Kubaje A, Williams I, Lengeler C, Maude GH, Armah GE, Kajihara B, Adiamah JH, Smith PG: Impact of permethrin impregnated bednets on child mortality in Kassena-Nankana district, Ghana: a randomised controlled trial. Trop Med Int Health 1996, I:147-154.

4. Gilles MT, De Meillon B: The Anophelinae of Africa South of Sahara (Ethiopian Zoogeographical Region). Publication of the South African Institute for Medical Research 2nd edition. 1968:343.

5. World Health Organization: Instructions for determining the susceptibility or resistance of mosquito larvae to insecticides. WHO/VBC/8I.807 1981:6. 
6. Fanello C, Petrarca V, Della Torre A, Santolamazza F, Dolo M, Coulibaly G, Alloueche A, Curtis CG, Toure YT, Coluzzi M: The pyrethroid knock-down resistance gene in the Anopheles gambiae complex in Mali and further indication of incipient speciation within An. gambiae s.s. Insect Mol Biol 2003, I 2:24I-245.

7. Ratovonjato J, Le Goff G, Rajaonarivelo E, Rakotondraibe EM, Robert $\mathrm{V}$ : Recent observations on the sensitivity to pyrethroids and DDT of Anopheles arabiensis and Anopheles funestus in the central Highlands of Madagascar; preliminary results on the absence of the kdr mutation in An. arabiensis. Archives de l'Institut Pasteur de Madagascar 2003, 69:63-99.

8. Elissa N, Mouchet J, Rivière F, Meunier JY, Yao K: Susceptibility of Anopheles gambiae to insecticides in the Ivory Coast. Sante 1994, 4:95-99.

9. Akogbéto $M$, Yakoubou S: Résistance des vecteurs du paludisme vis à vis des pyréthrinoödes utilisés pour l'imprégnation des moustiquaires au Bénin, Afrique de l'Ouest. Bull Soc Path Exot 1999, 92:123-130.

10. Diabate A, Baldet T, Chandre F, Akogbéto M, Guiguemde RT, Darriet F, Brengues C, Guillet P, Hemingway J, Graham JS, Hougard JM: The role of agricultural use of insecticides in resistance to pyrethroids in Anopheles gambiae s.l. in Burkina Faso. Am J Trop Med Hyg 2002, 67:617-622.

II. Corbel V, N'Guessan R, Brengues C, Chandre F, Djogbenou L, Martin $T$, Akogbéto M, Hougard JM, Rowland M: Multiple insecticide resistance mechanisms in Anopheles gambiae and Culex quinquefasciatus from Benin, West Africa. Acta Trop 2007, I0I:207-216.

12. Appawu MA, Baffoe-Wilmot A, Afari EA, Nkrumah FK, Petrarca V: Species composition and inversion polymorphism of the Anopheles gambiae complex in some sites of Ghana, West Africa. Acta Trop 1994, 56:15-23.

13. Wondji C, Simard F, Fontenille D: Evidence for genetic differentiation between the molecular forms $M$ and $S$ within the Forest chromosomal form of Anopheles gambiae in an area of sympatry. Insect Mol Biol 2002, I I: I I-I9.

14. Brown AWA, Pal R: Resistance des arthropods aux insecticides. Geneva. World Health Organisation, (WHO Monograph Series, No. 38); 1971.

15. Bruce-Chwatt LJ: Lessons learned from applied field research activities in Africa during the malaria eradication era. Bull World Health Organ 1984, 62:19-29.

16. Elissa N, Mouchet J, Rivière F, Meunier JY, Yao K: Resistance of Anopheles gambiae s.s. to pyrethroids in Côte d'Ivoire. Ann Soc Belge Med Trop 1993, 73:291-294.

17. Vulule JM, Beach RF, Atieli FK, MCallister JC, Brogdon WG, Roberts JM, Mwangi RW, Hawley WA: Elevated oxidase and esterase levels associated with permethrin tolerance in Anopheles gambiae from Kenyan villages using permethrin-impregnated nets. Med Vet Entomol 1999, 3:239-244.

18. Awolola TS, Brooke BD, Koekemoer LL, Coetzee M: Resistance of the malaria vector Anopheles gambiae s.s. to pyrethroid insecticides, in south-western Nigeria. Ann Trop Med Parasitol 2002, 96:849-852.

19. Etang J, Manga L, Chandre F, Guillet P, Fondjo E, Mimpfoundi R, Toto JC, Fontenille D: Insecticide susceptibility status of Anopheles gambiae s.l. (Diptera: Culicidae) in the Republic of Cameroon. J Med Entomol 2003, 40:49|-497.

20. Sharp BL, Ridl FC, Govender D, Kuklinski J, Kleinschmidt I: Malaria vector control by indoor residual insecticide spraying on the tropical island of Bioko, Equatorial Guinea. Malar J 2007, 6:52.

21. Appawu M, Owusu-Agyei S, Dadzie S, Asoala V, Anto F, Koram K, Rogers W, Nkrumah F, Hoffman SL, Fryauff DJ: Malaria transmission dynamics at a site in northern Ghana proposed for testing malaria vaccines. Trop Med Int Health 2004, 9: I64-I70.

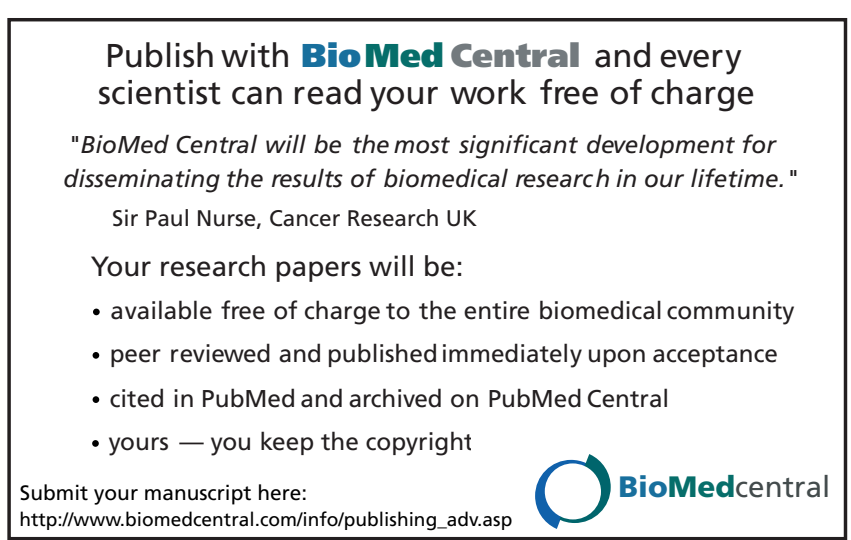

